\title{
Flipping the script
}

\author{
Joseph Lerro* \\ Routledge, Taylor \& Francis Group, 530 Walnut Street, Suite 850, Philadelphia, PA 19106, USA
}

\begin{abstract}
The global Open Access movement has undertaken a proposal to 'flip' from the traditional subscription model to an open access model. With so many stakeholders involved, such a transition must appeal to the interests of researchers, librarians, funders, and publishers. Likewise, with an array of open access models, it is important to determine which will be the most effective solution in the long term. Taylor \& Francis is taking a flexible, evidence-based approach to this transition, piloting a variety of models.

In addition to converting twenty-eight subscription journals to Full Open Access since January 2017, Taylor \& Francis has established open access agreements with organizations such as the Max Planck Digital Library and VSNU Dutch Library Consortium. This article will detail some of the publisher's experiences in flipping journals from hybrid to full open access and will provide specific examples of the effects and implications of transitioning to Open Access for a global publisher.
\end{abstract}

Keywords: Open access, publishing, business models, flipping, Taylor and Francis, scholarly journals

\section{Conversion to open access journals}

"Subscription journals have been converting or "flipping" to open access (OA) for about as long as OA has been an option [1]."

The above is a quote from Peter Suber in the Harvard conversion report Converting Scholarly Journals to Open Access: A Review of Approaches and Experiences. He goes on to say, "For just as long, OA proponents have been writing arguments on why to flip, recommendations on how to flip, and case studies on individual cases of flipping. But until now, no systematic study has reviewed the literature on journal flipping or distinguished the different pathways, methods, or scenarios for journal flipping [2]."

One of the key takeaways from the Harvard report is that there are a number of different models for conversion, not just moving to an Article Processing Charge (APC) model. Taylor \& Francis have embraced these options and have converted a number of society-owned journals to non-APC models. However, the conversion project being discussed here has focused on APC models.

Taylor \& Francis has been converting journals to Open Access since 2012, beginning with small titles in the physical sciences: Green Chemistry Letters and Reviews, Journal of Biological Dynamics, and International Journal of Smart and Nano Materials. This organic approach led to fifteen titles being converted between 2012 and 2016. In 2016 we launched the Conversion Project which saw twenty journals converted to Open Access for 2017 and seven conversions for 2018. Our conversion project goals are to

\footnotetext{
*E-mail: joseph.lerro@taylorandfrancis.com.
} 
accelerate Open Access conversion by:

- Establishing criteria for selection.

- Identifying more journals to convert each year.

- Completing the practical steps required to convert the group of journals AND establish Best Practice conversion processes.

- Collecting and analyzing data about the conversion journals to inform future strategy.

The project was inspired by our Open Access Vision, which is to "make it as easy as possible for researchers, societies, institutions, funders, and governments to achieve their open scholarship aims."

The scholarly landscape is in a state of transformation, and Taylor \& Francis is transforming along with it while also trying to contribute to this transformation. The organization wishes to enhance our reputation as a quality Open Access publisher with funders, institutions, policy makers, and research communities. By continuing to invest in Open Access and converting successful Taylor \& Francis journals to this business model, we make a statement on the future of Open Access publishing.

Our organization serves the open scholarship goals of diverse communities of researchers by offering both hybrid and full Open Access options. Researchers need more high quality Open Access journals covering specialized subjects; journals that meet the assessment needs of their discipline and their Open Scholarship goals; and full Open Access venues with a trusted reputation from a publisher who is a member of the Open Access Scholarly Publishers Association (OASPA - see: https://oaspa.org/) and journals included in the Directory of Open Access Journals (DOAJ - see: https://doaj.org/).

\section{Effects of the conversion}

- In January 2017 the archives dating back to 2007 were converted to free access for the twenty conversion project journals. Usage to the journals rose dramatically.

- Across the project as a whole there was a 247\% growth in usage through September 2017.

- Usage of articles in Pharmaceutical Biology rose from 46,894 to 365,026, a 678\% growth.

- There has been a great deal of variability in submissions figures, with many journals seeing an initial decrease in submissions. However, Journal of Freshwater Ecology has actually seen a 9\% growth of submissions since conversion.

- A rise in the quality of submissions has resulted in happy editors and reviewers spending less time on unpublishable submissions.

- It is still too soon to tell the effect conversion will have on revenue. Thus far, all the conversion project journals have dropped in revenue, which is something that had been expected.

The conclusion to Suber's preface to the Harvard conversion report implies that it is the publishers who need to be convinced about the potential for conversion, and editors must be the ones to perform that advocacy. Of course there are internal colleagues who have needed convincing, but our overall experience has been the other way around.

Each decision to convert a journal requires complex conversations with multiple stakeholders. The support and advocacy of editors and editorial boards is essential for success. It is fair to say that many more journals probably would have been converted had the editors and editorial board members been effectively convinced that such a conversion is in the best interest of the journals for which they are responsible. 
Some of their concerns?

- Impact Factor

- Ability to commission

- Concern about funding available to authors

- Ability to publish in their own journal.

Taylor \& Francis as a company has always prided itself on collaborative relationships with its editors and editorial boards and therefore pushing a journal into conversion without the support of the editorial team goes against the organization's culture. Success also depends upon an enthused and engaged board, so we prefer to work on advocacy with editorial groups where we see the potential for conversion. It can make conversion a long term goal though!

Perhaps the biggest lesson is 'flipping' a journal to Open Access is less conversion and more "rebirth." With a new publishing model comes the potential for new communities of authors and readers to gather around it. It is important for our editors and colleagues to see themselves as Open Access journals, compare themselves to other Open Access journals, and not constantly compare themselves to the pre-conversion incarnation of the journal. It offers the opportunity for a new identity. However, this is also the biggest challenge is leading colleagues and editors into unknown territory.

\section{About the Author}

Joseph Lerro is the Open Access Journals Sales Executive for the Americas at Taylor \& Francis. He works as part of a global team to develop and promote open access solutions for academic researchers and institutions. Email: joseph.lerro@taylorandfrancis.com.

\section{References}

[1] D.J. Solomon, M. Laakso and B. Björk, Converting Scholarly Journals to Open Access: A Review of Approaches and Experiences, P. Suber (ed.), Harvard University, 2016, p. 1 https://dash.harvard.edu/handle/1/27803834 (last accessed 14 June 2018).

[2] P. Suber, op. cit., p. 1, https://dash.harvard.edu/handle/1/27803834 (last accessed 14 June 2018). 